\title{
MEDICINAL MUSHROOM BIOACTIVES: POTENTIAL SOURCES FOR ANTI-CANCER DRUG DEVELOPMENT
}

\author{
KAINOOR K. JANARDHANANa, KORATTUVALAPPIL S. RAVIKUMARa, S. MOHAN KARUPPAYIL ${ }^{b}$
}

aAmala Cancer Research Centre, Amalanagar, Thrissur 680555 India, bDepartment of Stem Cell and Regenerative Medicine and Medical Biotechnology, DY Patil Education Institute (Deemed to be University), NACC Accredited With a Grade. Kolhapur, Maharashtra, India

Email: kkjanardhanan@yahoo.com

Received: 22 Jul 2020, Revised and Accepted: 10 Aug 2020

\begin{abstract}
Mushrooms represent a major yet largely untapped source of therapeutically useful bioactive compounds. Despite mushrooms were in use since antiquity in traditional folk medicine attempts to isolate their bioactive components and to elucidate their medicinal properties have started only recently. Many pharmaceutical substances with unique properties were recently extracted from mushrooms and made their way all over the world. A number of medicinal mushrooms have been identified to possess anticancer effects recently. Some of the well-known examples are Lentinan from Lentinus edodes, Krestin from Trametes versicolor, Ganopoly from Ganoderma lucidum and Schizophyllan from Schizophyllum commune. We investigated the anticancer activities of a number of medicinal mushrooms in our laboratory. Some of the recent scientific evdences on the anticancer activities of Ganoderma lucidum, Phellinus rimosus, and Fomitopsis pinicola are discussed in this short review.
\end{abstract}

Keywords: Medicinal mushroom, Drug development

(c) 2020 The Authors. Published by Innovare Academic Sciences Pvt Ltd. This is an open access article under the CC BY license (http://creativecommons.org/licenses/by/4.0/) DOI: http://dx.doi.org/10.22159/ijap.2020.v12s4.40103. Journal homepage: https://innovareacademics.in/journals/index.php/ijap

\section{INTRODUCTION}

According to World Health Organization (WHO) cancer is the second major cause of morbidity and mortality claiming over 9.6 million lives worldwide every year. Although significant contributions have been made on our understanding on the biology of cancer, discovery of anticancer drugs has been an extraordinary challenge. Thus, anticancer drug development remains one of the target areas of biomedical research. Despite the phenomenal progress in synthetic drugs, natural products still remain a major source for large number drugs. Hence, attention has now been focused on natural products for anticancer drug discovery. Despite the tremendous progress in modern medicine with the phenomenal developments in synthetic medicine, plants and their derivatives continue to be important sources of drugs throughout the world. Many clinically important drugs such as aspirin, digitoxin, progesterone, cortisones, morphine, vincristine, vinblastine, placitaxel (taxol) and several others have been derived directly or indirectly from higher plants.

Natural product-based medicines have originated from various sources, including plants, fungi and animals. Bioactive substances from these sources possess enormous structural and chemical diversity. Fungi have contributed enormously to modern medicine. A number of life-saving drugs such as penicillin, cyclosporine, ergot alkaloids and many others were developed from fungi. However, these drugs were all isolated and developed from micro-fungi. Mushrooms are macrofungi. The number of mushroom species on the earth is estimated to be 140,000 and currently, 14,000 to 15,000 species are known of which over 1800 species are considered to have medicinal properties [1]. A large number of bioactive chemical compounds have been isolated from the fruiting bodies, mycelia and spores of mushrooms.

The use of mushrooms as food and their use in medicine has been mentioned in ancient medical treatise, Charak Samhitha as early as 3000 B C. Most of the traditional knowledge about mushrooms comes from Far East, China, Japan, Korea and the east of Russia [2]. However, mushrooms represent a major yet largely untapped source of pharmaceutical products. Many pharmaceutical substances with unique properties were recently extracted from mushrooms and made their way all over the world. Some of the most recently isolated and identified compounds from medicinal mushrooms have shown promising immunomodulatory, antitumor, cardioprotective, antiviral, antibacterial, antiparasitic, hepatoprotective, and antidiabetic properties. Modern scientific studies on medicinal mushrooms have expanded exponentially during the last two decades not only in Japan, Korea, and China but also in USA [3]

One of the most important medicinal properties of mushrooms that have attracted the attention of public in recent years is their anticancer properties. Ikekawa and his coworkers published one of the first scientific reports on the antitumor activity of fruiting bodies of mushrooms in 1969. Thereafter three major anticancer drugs were developed from medicinal mushrooms, Krestin from Coriolus versicolor, Lentinan from Lentinus edodes, Schizophyllan from Schizophyllum commune. All the three were $\beta$-glucan polysaccharides. Krestin was developed in Japan from the strain of Trametes (Coriolus) versicolor, whereas Polysaccharide-peptide (PSP) in China was developed from the cultured mycelium of another strain of this mushroom. Lentinan, Krestin (PSP) and Schizophyllan are approved in Japan as prescription drugs for the treatment of cancer [4].

Over the past two or three decades, scientific and medical studies in Japan, China, Korea and, more recently in United States have increasingly demonstrated the potent and unique properties of mushroom extracted compounds for the prevention and treatment of cancer. The ability of mushrooms to inhibit tumor growth and modulate immune functions was demonstrated by several experimental studies and soon it became obvious that the tumorinhibiting activity was contained in the polysaccharide fractions.

Several mushroom polysaccharides have proceeded through Phase 1, II and III clinical trials and are extensively and successfully used in Asian countries to treat various types of cancers [5]. Medicinal mushroom products, especially, polysaccharides were developed with clinical and commercial purpose, Krestin (PSK) and PSP (Polysaccharide-protein complex) from Trametes versicolor; Lentinan from Lentinus edodes, Schizophyllan (Sonifilan, Sizofiran or SPG) from Schizophyllum commune, D-fraction from Grifola frondosa, Befungin from Inonotus obliququus; Ganopoly from Ganoderma lucidum and many others [5].

Experimental studies carried out at Amala Cancer Research Centre have found that number of medicinal mushrooms occurring in India was found to possess antitumor activity in animal models. However, further experimental studies revealed significant anticancer activity of Ganoderma lucidum, Phellinus rimosus, Morchella species, Pleurotus species, Volvariella volvaceae and Fomitopsis pinicola. 


\section{Ganoderma lucidum (Fr.) P. Karst}

G. lucidum (Fr.) P. Karst (fig. 1), popularly known as Reishi or Ling Zhi has the longest historical use as medicine dating back to $4000 \mathrm{y}$. This mushroom is used in traditional Chinese and Japanese medicines. Reishi is known as 'Plant of immortality' and key ingredient in the 'Elixir of life'. Although there were several fungi in folklore medicine, G. lucidum was considered as sacred. In Chinese folklore, LinghZhi is considered a panacea for all types of diseases. A large number of medicinal properties have been reported for this mushroom. Species of Ganoderma have been reported to occur throughout the world. G. lucidum is found to occur widely in India, particularly in tropical area. Investigations carried out at Amala Cancer Research Centre showed that G. lucidum occurring tropical south India possessed significant antioxidant, anti-inflammatory, antinociceptive, antimutagenic, anticarcinogenic, antitumor, hepatoprotective, nephroprotective and cardioprotective propeties.

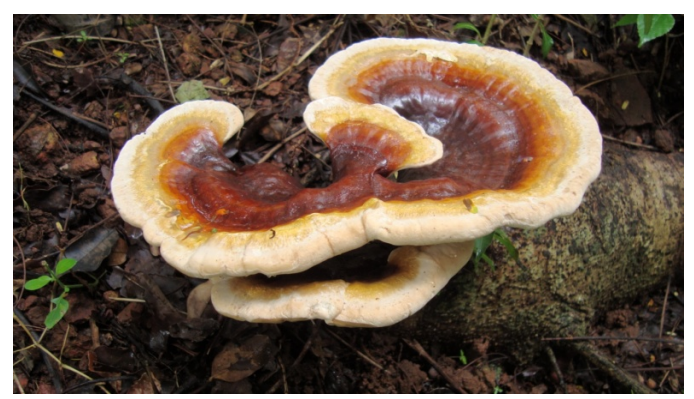

Fig. 1: Ganoderma lucidum

\section{Bioactive components}

A large number of bioactive chemical compounds were extracted from the fruiting body, mycelia and spores of Reishi-G. lucidum. Over 300 reports have been published on the chemical constituents of $G$. lucidum and related species. Approximately 400 bioactive compounds have been reported from the fruiting body, mycelium, and spores of $G$. lucidum. The bioactive molecules include polysaccharides, triterpenes, ganoderic acids, phenols, amino acids, lignin, vitamins, nucleosides, nucleotides, sterols, steroids, proteins, and unsaturated fatty acids, inorganic ions such as $\mathrm{Mg}, \mathrm{Ca}, \mathrm{Zn}, \mathrm{Mn}$, $\mathrm{Fe}, \mathrm{Cu}$ and $\mathrm{Ge}[6]$. However, polysaccharides and triterpenes are the two major groups of bioactive compounds present in G. lucidum [7].

Polysaccharides of Reishi are the most important component and represent structurally-diverse biological macromolecules with wide range of biological and physiochemical properties [8]. More than 200 polysaccharides, including protein-bound polysaccharides have been reported from $G$. lucidum. The major bioactive polysaccharides isolated from Ganoderma species are $\beta$ 1-3 and $\beta$ 1-6 D glucans (fig. 2 ) with molecular weight ranging from $4 \times 10^{5}$ to $1 \times 10^{6}$.

The second most important biologically active compound in Reishi is triterpenoids/triterpenes. This group has received considerable attention because of their well-known pharmacological activities. More than 130 oxygenated triterpenes (mostly lanostane-types) have been isolated till recently from the fruiting bodies, spores, mycelia, and culture media of Reishi-G. lucidum [9]. These triterpenes could be divided into $\mathrm{C} 30, \mathrm{C} 27$, and C24 compounds according to the number of carbon atoms and based on the structure and functional groups (fig. 3)). In general, they are low molecular weight compounds. These compounds isolated from Reishi were intensively studied during 1980s. Currently, nearly, 136 triterpenes with varying structures have been isolated [5].

\section{Anticancer activity}

Ganoderma species and their extracts are known as a traditional remedy in Chinese medicine for the prevention and treatment of cancer [10]. Numerous pharmacological investigations demonstrated that hot water extract of G. lucidum inhibited tumor growth in tumor-bearing mice. Several studies carried in our laboratory and elsewhere have demonstrated the significant antitumor effect of $G$. lucidum [11, 12]. The alcoholic extract has been demonstrated to possess a significant effect on mammary adinocarcinoma and skin papilloma [13].

The most important constituents of G. lucidum are terpenoids and polysaccharides. Both of these constituents show remarkable antitumor activity [14]. Triterpenes isolated from G. lucidum were found to have cytotoxic activity against several tumor cell lines. The alcohol extract of G. lucidum was shown that it inhibited cell proliferation in a dose and time-dependent manner. Triterpene fraction of G. lucidum significantly inhibited human hepatoma cells. Oxygenated triterpenes isolated from G. lucidum spores also showed direct cytotoxicity in vitro on tumor cell lines [15]. G. lucidum triterpenes were shown to possess profound activity against leukemia, lymphoma, and multiple myeloma.

The antitumor effect of G. lucidum was mediated by cytokines released from activated $\mathrm{T}$ lymphocytes and macrophages. The currently available information suggests that intrinsic immunological mechanisms are involved in the antitumor activity of G. lucidum. Antitumor effect also has been demonstrated by inhibiting angiogenesis. The angiogenesis potential might direct inhibition of vascular endothelial proliferation or decrease growth factor expression of tumor cells [16]. Clinical studies also showed that $G$. lucidum preparations exerted a synergistic therapeutic effect when used in conjunction with radiation and chemotherapy and also reduced the side effects of these treatments.

It is generally accepted that the antitumor and anticancer effects of Ganoderma polysaccharides arise from the enhancement of the body's immune system rather than direct cytotoxic effects. However, Reishi-G. lucidum was the most active cytotoxic to cancer cells among the large number of Basidomycetes species tested. Several studies correlated the tumoricidal effects of Reishi with the regulation of cancer cell cycling and signaling [17]. Recent preclinical studies undertaken in our laboratory showed that $G$. lucidum possessed a significant protective effect against mammary adenocarcinoma and skin tumors [13].

We carried out preclinical studies to find out the antitumor effect of polysaccharides and triterpenes isolated from the fruiting bodies of G. lucidum. We found that the polysaccharides inhibited $88 \%$ tumor growth at a dose of $25 \mathrm{mg} / \mathrm{kg}$ body weight and the triterpenes inhibited $85 \%$ tumor growth at a dose of $10 \mathrm{mg} / \mathrm{kg}$ body weight in treated animals. However, polysaccharides and triterpenes isolated from the mycelia showed higher antitumor activity than fruiting bodies. Total terpenes induced apoptosis in MCF-7 cell lines and also inhibited 7,12, dimetylbenz [a] anthracene (DMBA) induced mammary adenocarcinoma and skin tumor [18].

Clinical observation assessment of 547 medium and late phase cancer patients treated with Chinese $G$. lucidum essence showed that the death rate of patients in long-term treatment was significantly low. A continuous 2-3 mo active treatment with a daily dose of 4-6 g G. lucidum essence was proposed. Two randomized trials using $G$. lucidum polysaccharide (Ganopoly) was reported [17]. A total of 34 advanced patients of different cancer origin were recruited and administered $12 \mathrm{w}$ with Reishi capsules at a dose of $1800 \mathrm{mg}$ per day and $80 \%$ of patients were found to have a series of immunological enhancements. In another study, the same capsules were administered to 68 lung cancer patients and over $65 \%$ of the patients were found to have improvements in quality of life. Based on available consolidated scientific evidence and clinical observations Red mushroom, G. lucidum is highly beneficial to cancer patients [10].

In a phase I study, methanol extract of G. lucidum was administered to men with mild symptoms of bladder outlet obstruction. Male volunteers of age above 50 were enrolled. They observed a statistically significant reduction in prostrate symptoms at doses of 6 to $60 \mathrm{mg}$ daily and recommended phase 2 trails. Two randomized clinical trials were reported by Gao and coworkers [17]. A total of 34 cancer patients were recruited, and 12-week Reishi capsules at a dose of $1800 \mathrm{mg}$ were given and $80 \%$ of the patients were observed 
significant relief. In another study of 68 lung cancer patients, improvement in qualities of life of $65 \%$ patients was observed.

Current experimental evidence indicates that $G$. lucidum can inhibit the signaling pathway responsible for increased migratory potential of cancer cells. This process has clinical importance and inhibiting the motility of cancer cells can prevent metastatic spread of cancer into distant tissues of human body. Angiogenesis is essential for invasiveness and metastasis of tumors and accumulating evidence indicates that inhibition of angiogenesis is an effective cancer preventive and treatment. Antiangiogenisis properties are believed to be one of the many possible cancer preventive mechanisms. Hence Reishi can be considered as an adjuvant in cancer treatment [15]

Almost all toxicity studies with Ganoderma using animal models showed a low or no detectable toxicity. There are no reported data on long term adverse effects on G. lucidum and its derivatives. The aqueous extract of Reishi administered to mice orally at a dose of $5 \mathrm{~g} / \mathrm{kg}$ body weight for $30 \mathrm{~d}$ produced no changes in body weight, organ weight, or hematological parameters. The polysaccharides fraction at the same dose produced no lethal or serious effects. No toxicity was observe in rats, dogs and rabbits [19].

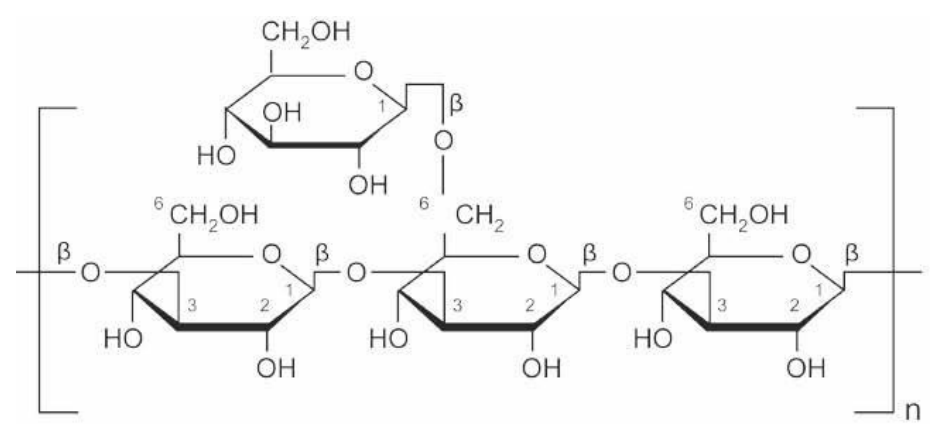

Fig. 2: Structure of Ganoderma polysaccharides (Real mushroom. com)

Among the various classes of chemical constituents isolated from $G$. lucidum Polysaccharides $(\beta-1 \rightarrow 3$ and $\beta-1 \rightarrow 6-\mathrm{D}$ glucans $)$ and triterpenoids such as Ganoderic acids are the major compounds showing antitumor properties.

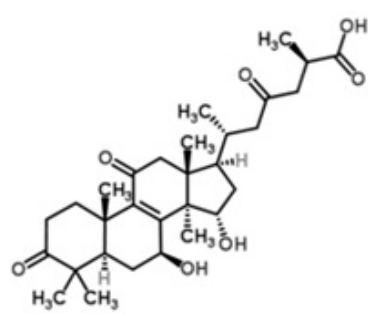

Ganoderic acid A

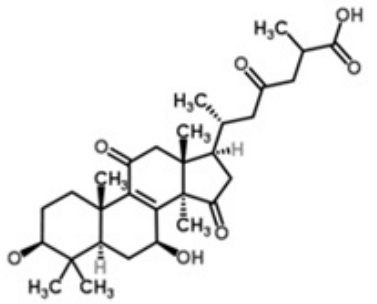

Ganoderic acid B

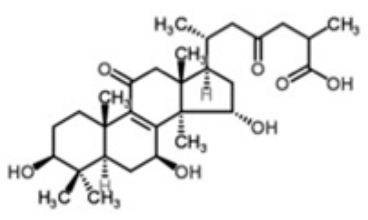

Ganoderic acid B

Fig. 3: Basic structure of main Ganoderma triterpenes, (Benkeblia, N J. Food. Nutri. Diete, 2015)

\section{Phellinus rimosus (Berk) pilat}

Approximately 220 species of Phellinus are known. In traditional Chinese medicine hot water extracts of $P$. linteus have been used to treat extensive range of ailments and it is believed to work as a 'miracle medicine. Recent studies showed that $P$. linteus extract possessed strong antiproliferative effect [20]. It has been extensively studied for the effect on gastric, duodenal, colon, rectal and liver cancers. Isolated compounds from cultured mycelia of Phellinus species have been used in Korea as medicine, particularly for cancer treatment. $P$. linteus showed antitumor properties owing to its $\beta$ - $(1$ 3) linked glycan.

Polysaccharides isolated from $P$. gilvus significantly inhibited benzo $[a]$ pyrene-induced forestomach carcinogenesis in mice by down-regulating p53 expression. P. linteus is a well-known species of the genus Phellinus, which attracted great attention due to its potent anti-tumor effect and other medicinal properties [21]. The anti-tumor activity of the polysaccharides from the fruiting body of this mushroom was first reported in 1968, thereafter a wide variety of reports have been documented by many investigators. In a comparative study of polysaccharides from Basidiomycetes, the polysaccharide of $P$. linteus was the most potent one and produced growth inhibition of $96.7 \%$ in Sarcoma 180 transplanted immunocompetent ICR mice. The active polysaccharide purified from mycelia culture of $P$. linteus also stimulates humoral and cell-mediated immunity, and exhibited a wider range of immunostimulation and anti-tumor activity than other polysaccharides isolated from Basidiomycetes. Phellinus polysaccharide was also suggested to be used in immunochemotherapy of cancer because of its effectiveness on tumor growth and metastasis through the immunopotentiation without toxicity. Many investigators have been demonstrated that the mechanism of anti-tumor action of Phellinus mushroom involves multiple processes Results of the recent study suggest that the biological response modifiers that stimulates proliferation and expression of co-stimulatory molecules in B cells, probably by regulating protein tyrosine kinase (PTK) and protein kinase $C$ (PKC) signaling pathways [22]. Another study showed that the tumoricidal activity of peritoneal macrophages against B16 melanoma cells was enhanced in a dose-dependent manner. This study concluded that Phellius linteus act as an effective immunomodulator and enhances the anti-tumoral activity through the up-regulation of nitric oxide (NO) and Tumor necrosis factor- $\alpha$ (TNF- $\alpha)$. Extract of $P$. linteus was reported to possess antimutagenic activities and play a role in the prevention of cancer by inducing $\mathrm{NAD}(\mathrm{P}) \mathrm{H}$ : quinine oxidoreductase and glutathione S-transferase activities and increasing glutathione level.

Direct cytotoxicity of $P$. linteus against cancer cells inhibits the cellular proliferation in human colon cancer cells by induction of apoptosis 
and G2/M phase arrest. Hispolon (fig. 4), a phenolic compound isolated from this mushroom has potential to induce apoptosis of breast cancer cells. These findings indicate the potential use of the $P$. linteus extracts in stimulated angiogenesis indicating potential therapeutic approach as an adjuvant for chemotherapy [23].<smiles>CC(=O)/C=C(O)/C=C/c1ccc(O)c(O)c1</smiles>

Fig. 4: Hispolon-a bioactive anticancer compound isolated from Phellinus species

Phellinus species are mostly tropical mushrooms and 18 species are known from Kerala, India. P. rimosus is a parasitic macrofungus that grows on jackfruit trees in Kerala (fig. 5). Basidiocarps of this mushroom have been used by some tribes in Kerala for curing mumps [24]. Intensive investigations were carried out in our laboratory on the therapeutic potential of this mushroom. Our studies have demonstrated that $P$. rimosus possessed significant antioxidant, anti-inflammatory, antiarthritic, antitumor, chemopreventive, hepatoprotective, and radioprotective activities.

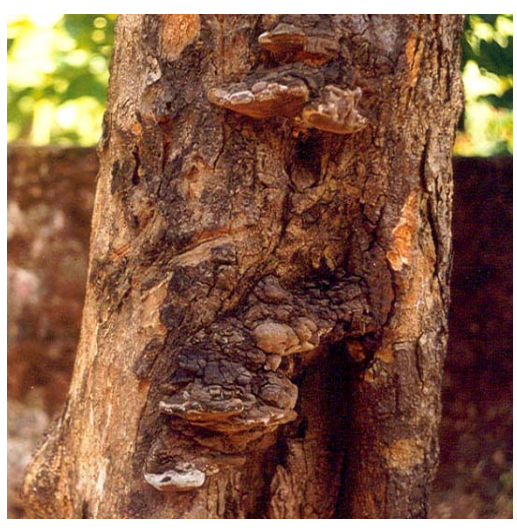

Fig. 5: Phellinus rimosus

The methanol and ethyl acetate extracts of P. rimosus showed significant superoxide anion, hydroxy radical, and nitric oxide scavenging and lipid peroxidation inhibiting activities [25]. Ethyl acetate and methanol extracts also showed significant cytotoxicity against Dalton's lymphoma ascites (DLA) and Earlich's ascites carcinoma cell lines (EAC). Antitumor activity of the ethyl acetate and methanol extracts showed significant antitumor inhibiting activity in animal mode [26]. The ethyl acetate extract also showed profound anti-mutagenic activity which can be ascribed to its mechanism of antitumor activity [27]. Meera and Janardhanan [28] reported the isolation of a crude polysaccharide bound protein from fruiting bodies of $P$. rimosus (PPC-Pr). The polysaccharide-protein was found significant preventive and curative effects on solid tumors in animal models. The aqueous extract of $P$. rimosus was observed significant chemopreventive activity against $\mathrm{N}$ nitrosodiethylamine (NDEA) induced hepatocellular carcinoma [29].

The phytochemical analysis revealed that PPC- $P r$ is a D-glucanprotein complex with a molecular weight $1,200,000 \mathrm{Da}$. Anti-tumor activity of PPC-Pr was evaluated using the EAC and DLA murine cell lines. PPC-Pr administration considerably increased the life span of EAC induced ascetic tumor bearing mice. PPC-Pr also showed significant preventive and curative effects on solid tumor induced by DLA cell line.

Apoptosis plays an important role as a protective mechanism against carcinogenesis by eliminating damaged cells or abnormal excess cells proliferation owing to various chemical agents' induction. Emerging evidence has demonstrated that the anti-cancer activities of certain chemotherapeutic agents are involved in the induction of apoptosis, which is regarded as the preferred way to manage cancer. PPC-Pr was able to induce significant cytotoxicity against HCT 116 colon cancer cell line. PPC-Pr inhibited the proliferation of HCT 116 cells markedly at a dose of 500 and $1000 \mu \mathrm{g} / \mathrm{ml}$. The characteristic changes of apoptosis were observed in the morphology of PPC-Pr treated cells by 4', 6-Diamidino-2phenylindole dihydrochloride hydrate (DAPI) and Acridine orangeethidium bromide (AO/EB) staining methods Chromatin degradation into multiple internucleosomal fragments is a distinct biochemical hallmark for apoptosis and that can be easily detected by Comet assay. Comet assay revealed that the apoptotic nuclei were more frequent in PPC-Pr treated cells than the control. Thus, by long term incubation, PPC-Pr induced cytotoxicity and inhibited the tumor cell proliferation in vitro by the induction of apoptosis [30]. These studies indicated the potential therapeutic use of $P$. rimosus as a potent anticancer agent.

\section{Fomitopsis pinicola(Sw) P. Karst}

Fomitopsisis is a genus having more than 40 species of bracket fungi in the family fomitopsidaceae. Fomitopsis species have fruit bodies that are mostly perennial, with forms ranging from sessile to effused-reflexed (partially crust-like and partially pileate). Fruiting body texture is typically tough to woody, and the pore surface is white to tan or pinkish-colored with mostly small and regular pores. Non-toxic Fomitopsis has been traditionally used in folk medicine in many countries. The fungus was widely used in traditional European medicine.

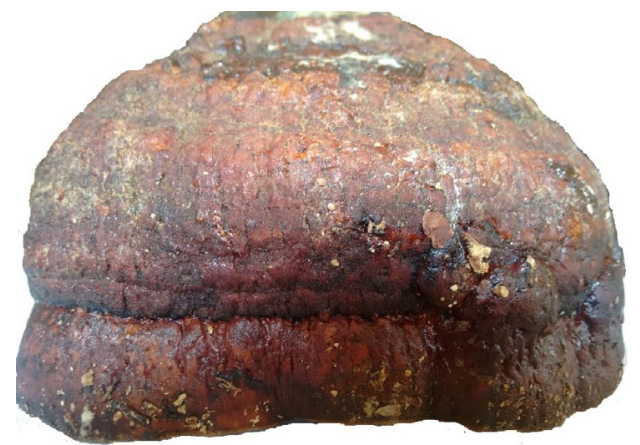

Fig. 6: Fomitopsis pinicola collected from kashmir conifer forest

F. pinicola is a common mushroom which grows on trees in coniferous forests. The mushroom is used for medicinal purposes in Asia and reported to provide health, nutrition, and therapeutic benefits. Fomitopsis has been reported to contain several chemical constituents including ergosterol, phenolics, triterpenes, picolinic acid and many other compounds. It has been used in folk medicine as antiiinflammatory, antimicrobial, and anticancer agents. Chemical compounds reported from $F$. pinicola include steroids, sesquiterpenes, lanostane triterpenoids and triterpene glycosides [31].

We investigated the antioxidant, anti-inflammatory and anticancer properties of $F$. pinicola collected from Kashmir Himalayas (fig. 6). Ethyl acetate and ethanol extracts showed significant antioxidant, anti-inflammatory and antitumor activities. The extract also showed significant activity against 7, 12 Dimethyl benz [a] anthracene (DMBA) induced cancerous papilloma.

MTT assay showed that ethyl acetate extract was highly effective in killing the DLA cells. Even at a low concentration of $50 \mu \mathrm{g} / \mathrm{ml}$, the extract caused significant cytotoxicity. Angiogenesis, the process for 
the production of a large number of capillaries from the blood vessels is an important property of malignant cells. Angiogenesis is a multi-step process that is important for the establishment of cancer growth. Ethyl acetate extract of $F$. pinicola showed significant antiangiogenic property. The extract induced high amount apoptotic activity in human colon cancer cell line (HCT 116). These studies showed the anticancer effect of $F$. pinicola bioactives [32].

A literature survey reveal that a large number of studies on the anticancer activity of $F$. pinicola have been carried out using cell cultures. Wang et al. [33] isolated ergosterol (fig. 7) from the chloroform extract of $F$. pinicola. They observed anticancer properties such as pro-apoptotic and inhibition of migration effect of this compound. However, further studies are required to characterize the anticancer effect of $F$. pinicola. Such investigations would be interesting in order to ascertain the therapeutic benefits of this mushroom as an anticancer agent [34]. The added advantage is that $F$. pinicola has been reported nontoxic by anecdotal evidences and has been popular in folklore medicine.

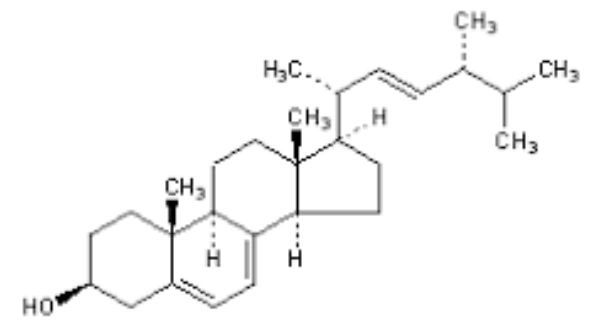

Fig. 7: Ergosterol-bioactive anticancer compound reported from F. pinicola

\section{Future prospects}

Large majority of anticancer drugs available today are generated from natural products. However mushrooms remain largely unexplored source for drug discovery although they have been demonstrated to possess significant antitumor, immunomodulating, cytotoxic and antiangiogenic properties. Over the last two decades scientific and medical research in Japan, Korea, and recently in United States have demonstrated the potent therapeutic properties of mushroom bioactives for the prevention and treatment of cancer. Some of the most effective antitumor compounds from mushrooms included,Krestin from Trametes vesicolor, Lentinan from Lenitnus edodes, Schizophyllan from Schizophyllum commune, Grifolan from Grifola frondosa, SSG from Sclerotinia sclerotiorum and Ganopoly from Ganoderma lucidum. Krestin, Lentinan and Schiziphyllan are prescription drugs for treatment of cancer in China and Japan[5]. Experimental studies carried out by us at Amala Cancer Research Centre found that several mushrooms, namely, G. lucidum, $P$. rimosus, Pleurotus florida, Pleurotus sajor-caju, Morchella esculenta, Volvariella volvaceae and F. pinicola possessed profound antitumor properties having potential to develop as anticancer agents. Anticancer bioactives isolated from medicinal mushrooms include polysaccharides, protein bound polysaccharides, steroids, sesquiterpenes, lanostane triterpenoids, triterpene glycosides, phenolic compounds etc. The current experimental studies worldwide reveal the possibilities of several mushroom bioactives for the development of anticancer agents or drugs especially as adjuvant cancer therapy. They have been suggested to enhance the efficacy of treatment and for the alleviation of side effects of chemo and radiation therapy [35]. Moreover, numerous clinical trials were conducted in recent years to assess the clinical use of a number of medicinal mushrooms. The research achievements during the last two decades demonstrated that medicinal mushrooms have much to offer for the development of anticancer drugs.

\section{FUNDING}

Nil

\section{AUTHORS CONTRIBUTIONS}

All the authors have contributed equally.

\section{CONFLICT OF INTERESTS}

\section{Declared none}

\section{REFERENCES}

1. Chang ST, Miles P. Mushrooms, cultivation, nutritional value, medicinal effects and environmental impact CRC Press: New York, USA; 2004.

2. Hobbs Ch. Medicinal mushrooms: an exploration of tradition, healing and culture. $2^{\text {nd }}$ Ed. Botanica Press: New York, USA; 1995.

3. Janardhanan KK, Ajith TA. Developments in medicinal mushrooms: biology and therapeutic properties. Astral International Pvt. Ltd, New Delhi, India; 2015. p. 19-34.

4. Smith J, Rowan N, Sullivan R. Medicinal mushrooms: their therapeutic properties and current medicinal usages with special emphasis on cancer treatment. Special report commissioned by Cancer Research UK; The University of Strathelyde in Glasgow; 2001.

5. Wasser SP. Current findings, future trends and unsolved problems in studies of medicinal mushrooms. Appl Microbiol Biotechnol 2010;89:1323-32.

6. Zhou X, Lin J, Yin Y, Zhao J, Sun X, Tang K. Ganodermataceae: natural products and their related pharmacological functions. Am J Chinese Med 2007;35:559-74.

7. Wasser SP. Reishi or ling zhi (Ganoderma lucidum)) encyclopedia diet supplement; 2005. p. 603-22.

8. Bao XF, Liu CP, Fang JN, Li XY. Structural and immunological studies of a major polysaccharide from spores of Ganoderma lucidum (Fr.) P. Karst. Cabohydrate Res 2001;332:67-74.

9. Kim HW, Kim BK. Biomedical tritepenoids from Ganoderma lucidum (Curt. :Fr.) P. Karst (Aphyllophoromycetidaae). Int J Med Mushr 1999;1:121-38.

10. Yuen TWM, Gohel MDI. Anticancer effects of Ganoderma lucidum: review of scientific evidence. Nutr Cancer 2005;53:11-7.

11. Jones S, Janardhanan KK. Antioxidant and antitumor activity of Ganoderma lucidum (Curt.: Fr) P. Karst.-Reishi (Aphyllophoromycetideae) from South India. Inter J Med Mushrooms 2000;2:195-200.

12. Jin X, Ruiz Beguerie J, Sze DM, Chan GC. Ganoderma lucidum (Reishi mushroom) for cancer treatment. Cochrane Database Syst Rev 2016;4:CD007731.

13. Lakshmi B, Sheena N, Janardhanan KK. Prevention of mammary adenocarcinoma and skin tumor by Ganoderma lucidum, a medicinal mushroom occurring in South India. Cur Sci 2009;97:1658-63.

14. Joseph S, Sabulal B, George V, Antony KA, Janardhanan KK. Antitumor and anti-inflammatory activities of polysaccharides isolated from Ganoderma lucidum. Acta Pharm 2011;61:335-42.

15. Sliva D. Ganoderma lucidum (Reishi) in cancer treatment. Integ Cancer Ther 2003;2:358-64.

16. Zang NM, Zang L. Isolation, purification and pharmacological actions and application to functional food of Ganoderma lucidum polysaccharide. Food Res Dev 2005;26:118-20.

17. Gao Y, Zhou S, Jiang W, Huang M, Dai X. Effect of ganopoly (a Ganoderma lucidum polysaccharide extract) on the immune functions in advanced-stage cancer patients. Immunol Inves 2003;32:201-15.

18. Smina TP, Nith B, Devasagayam TPA, Janardhanan KK. Ganoderma lucidum total terpenes induce apoptosis in MCF-7 cells and attenuate DMBA induced mammary and skin carcinoma in experimental animals. Mutation RE/Genetic Tox Environ Mutag 2017;813:45-51.

19. Bishop KS, Kao CHK, Xu Y, Glucina MP, Paterson RRM. From 2000 y of Ganoderma lucidum to recent development. Phytochemistry 2015;114:56-65.

20. Mizno T. The extraction and development of antitumor active polysaccharides from medicinal mushroomsin Japan (review). Int J Med Mushrooms 1999;1:9-29. 
21. Konn S, Chu K, Feuer N, Phillips J, Choudhur M. Potent anticancer effects of bioactive mushroom extracts (Phellinus liteus) on a variety human cancer cells. J Clin Med Res 2015;7:76-82.

22. Zhu T, Guo J, Collins L, Kelly J, Xiao ZJ, Kim SH, et al. Phellinus linteus activate different pathways to induce apoptosis in prostate cancer cells. Br J Cancer 2007;96:583-90.

23. Sliva D. Medicinal mushroom, Phellinus linteus as an alternative cancer therapy (Review). Exp Ther Med 2010;1:407-11.

24. Ganesh PN. Studies on wood inhabiting macrofungi of Kerala. Ph. D Thesis, University of Calicut, India; 1988. p. 45.

25. Ajith TA, Janardhanan KK. Indian medicinal mushrooms as a source of antioxidant and antitumor agents. J Clin Biochem Nutr 2007;40:157-62.

26. Ajith TA, Janardhanan KK. Cytotoxic and antitumor activities of a polypore macrofungus, Phellinus rimosus (Berk) pilat. J Ethanopharmacol 2003;84:157-62.

27. Ajith TA, Janardhanan KK. Antimutagenic effect of Phellinus rimosus (Berk) Pilat against chemical induced mutation of histidine dependent Salmonella typhimurium strains. Food Chem Toxicol 2011;49:2676-80.

28. Meera CR, Janardhanan KK. Antitumor activity of a polysaccharide-protein complex isolated from a wood-rotting polypore macro fungus Phillinus rimosu s(Berk) Pilat. J Environ Path Oncol 2012;31:223-32.

29. Ajith TA, Janardhanan KK. Chemopreventive activity of macrofungus Phellinus rimosus against $\mathrm{N}$ nitrosodimethylamine induced hepatocellular carcinoma in rat. J Exp Ther Oncol 2006;5:1-13.

30. Meera CR, Janrdhanan KK, Karunagaran D. Antiproliferative and apoptotic activities of the medicinal mushroom Phellinus rimosus (Agaricomycetes) on HCT116 human colorectal carcinoma cells. Inter J Med. Mushrooms 2018;20:935-45.

31. Rosecke J, Koing W. Constuents of various wood-rotting basidomycetes. Phytochemistry 2000;54:747-50.

32. Cheng J, Lin C, Lur H, Chen H, Lu M. Properties and biological functions of polysaccharides and ethanol extracts isolated from medicinal fungus, Fomitopsis pinicola. Proc Biochem 2008;43:829-34.

33. Wang, Cheng $\mathrm{X}$, Wang $\mathrm{P}$, Wang $\mathrm{L}$, Ran J, Wang $\mathrm{X}$, et al. Investigating migration and apoptotic effects of Fomitopsis pinicola chloroform extracton human colorectal cancer SW-480 cells. PloS One 2014;9:e101303.

34. Bishop KS. Characterization of extracts and anticancer activities of Fomitopsis pinicola. Nutrients 2020;12:609.

35. Patel S, Goyal A. Recent development in mushrooms as anticancer therapeutics: a review. 3 Biotech 2012;2:1-15. 